\title{
Single Electron Dynamics in Liquid-Gated Two-Layer Silicon Nanowire Structures: Towards Biosensing with Enhanced Sensitivity
}

\author{
Y.Kutovyi $^{1}$, I.Zadorozhnyi ${ }^{1}$, V.Handziuk ${ }^{1}$, N.Boichuck ${ }^{1}$, H.Hlukhova ${ }^{1}$, M.Petrychuk ${ }^{1,2}$ and \\ S.Vitusevich ${ }^{1}$ \\ ${ }^{1}$ Bioelectronics (ICS-8), Forschungszentrum Jülich \\ 52428 Jülich, Germany \\ Phone: +49-2461-61-2345 E-mail: s.vitusevich@fz-juelich.de \\ ${ }^{2}$ Faculty of Radiophysics, Electronics and Computer Systems \\ Taras Shevchenko National University of Kyiv \\ Kyiv, Ukraine
}

\begin{abstract}
We fabricated new types of nanowires that consist of two silicon layers with different doping concentrations. Fabricated structures demonstrate pronounced random telegraph signal fluctuations as a result of single electron dynamic processes. Liquid-gated nanowire transistor devices show advanced characteristics favorable for a new sensing approach based on single trap phenomena.
\end{abstract}

\section{Introduction}

The selective and highly-sensitive detection of biomolecules using biosensors based on silicon nanowire field-effect transistors (Si NW FETs) has been one of the top priorities in biosensing for over two decades. In order to achieve even higher sensitivity which allows for single molecule detection, nanowires should become shorter and narrower. However, when sensors are scaled down, the excess noise may result in serious limitations on the sensing performance. In particular the drain current fluctuations between several distinct levels can be registered in the time domain when nanowire channel is biased at a fixed potential. These fluctuations, also known as random telegraph signal (RTS) noise, reflect single charge carrier trapping by an active single trap in the gate dielectric layer near the semiconductor channel. Usually, such RTS noise is considered as an unwanted process, which strongly affects the useful sensory signal. However, the positive outcome of such current fluctuations has been recently demonstrated for $\mathrm{pH}$-sensing using liquid-gated Si NW FETs [1]. In particular, it was shown that changes of surface potential caused by changes in the $\mathrm{pH}$ of the gating solution can be registered with enhanced sensitivity by monitoring the charge carrier capture time as a novel sensing parameter. This approach was demonstrated to be more sensitive in comparison to the drain current changes.

In general, the dependence of capture time on the drain current in nanowire channel can be obtained by the statistical analyses of RTS measurements. According to conventional Shockley-Reed-Hall (SRH) theory it is linear because of the linear capture rate dependence on the carrier's concentration. However, experimentally it has been shown that the capture time characteristic behaves like the power function of drain current [1-4]:

$$
\tau_{c} \sim I_{D}^{-\gamma}
$$

where $\tau_{c}$ is the average capture time of single electron, $I_{D}$ is the drain current in the Si NW FET, $\gamma$ is an order of the power function. Obviously, that the efficiency of this new approach for biosensing, in comparison to the standard drain current change approach, strongly depends on the order of the power function $\gamma$. Usually, this parameter acquires different values from 1 to 5 for typical nanowire FETs [1-4] and depends on the carrier concentration in the channel.

Therefore, in order to achieve a better single trap switching kinetic, i.e. higher order of the power law, we have fabricated nanowires that consist of two silicon layers with different impurity concentrations: the first layer is top active silicon layer of silicon-on-insulator wafers with an impurity concentration of $10^{15} \mathrm{~cm}^{-3}$, while the second layer is highly-doped silicon with an impurity concentration of $10^{17} \mathrm{~cm}^{-3}$, which was epitaxially grown on the top of the first silicon layer.

\section{Results and discussion}

The structures under investigations are single nanowires, which are $100 \mathrm{~nm}$ wide and 200nm long, configured as liquidgated n-channel enhancement FETs. Typical high-resolution scanning electron microscope (SEM) images of well-defined nanowire structures are presented in Fig.1.

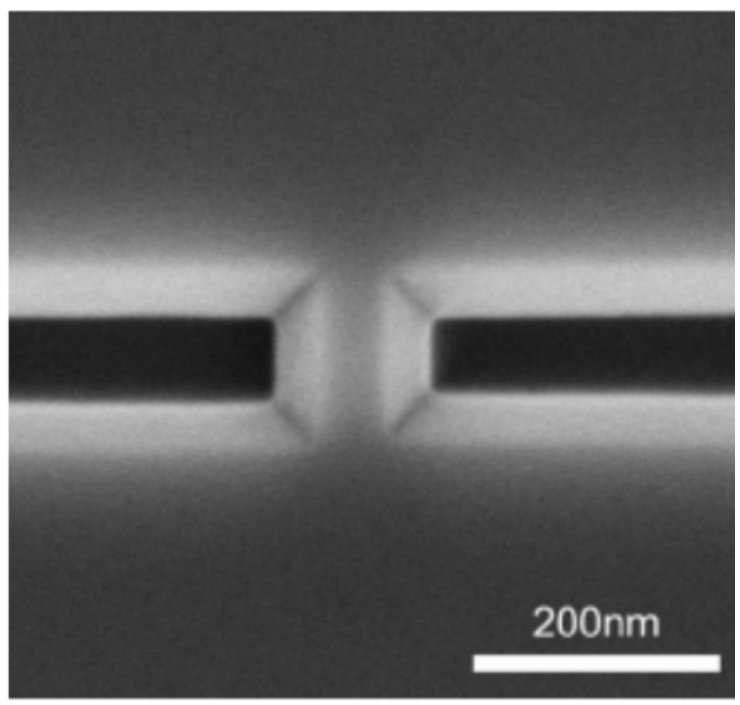

Fig.1. High-resolution SEM image of fabricated Si NW FET. 
In general, fabricated devices behave like conventional MOSFETs, yielding a Lorentzian shaped noise power spectral density (PSD) at room temperatures. Such noise behavior suggests the presence of single electrically active traps in the top gate dielectric layer which are responsible for the RTS noise.

A typical RTS noise PSD of a single nanowire transistor measured in $\mathrm{PBS}$ solutions with $\mathrm{pH}=7.4$ at constant liquidgate voltage of $1.8 \mathrm{~V}$ and drain-source bias of $100 \mathrm{mV}$ is presented in Fig.2. The associated pure two-level RTS signal with high amplitude in the time domain is depicted in the inset of the Fig.2.

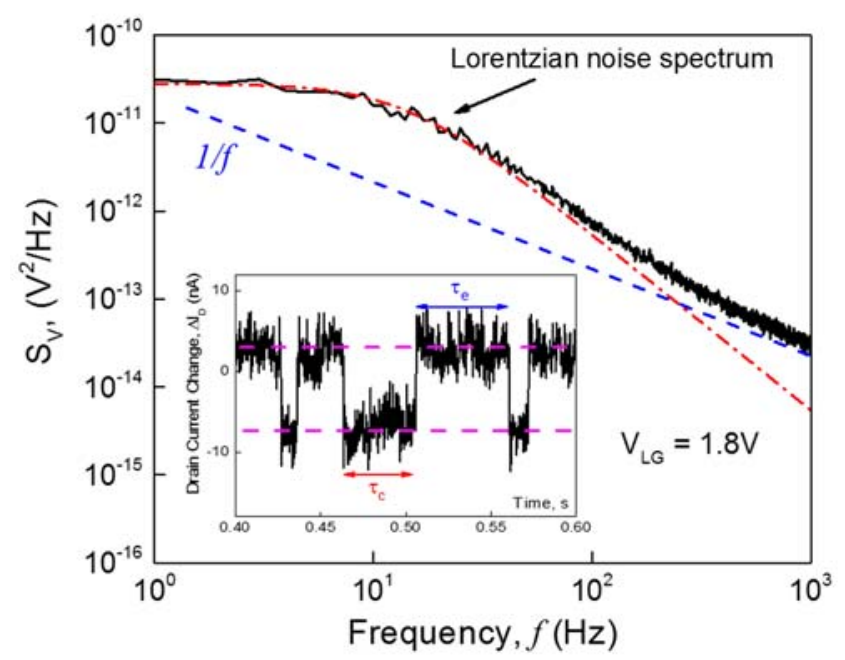

Fig.2. RTS noise PSD (Lorentzian shape - red line) and corresponding drain current fluctuations in liquid-gated $\mathrm{Si}$ NW FET.

In order to identify and analyze the RTS noise component from the inherent transistor noise, a statistical histogram method was used. As a result, the average electron characteristic time $\tau$, capture time $\tau_{c}$ and emission time $\tau_{e}$ were calculated and plotted as functions of drain current in Fig.3. The data demonstrates that the characteristic capture time depends on the drain current with a power of -6.25 , which goes far beyond the predictions of SRH model.

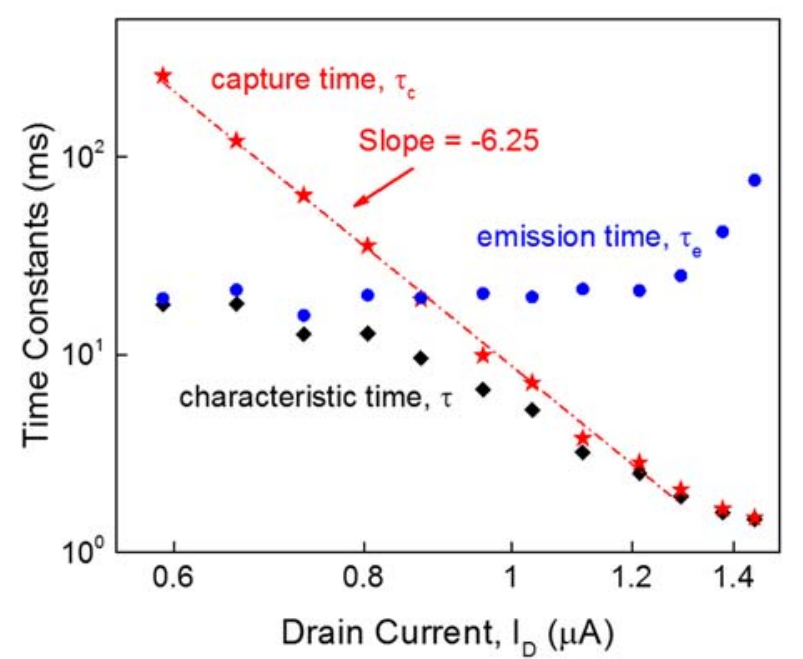

Fig.3. Time constants of the RTS noise plotted versus drain current. Dashed red line with slope (-6.25) reflects behavior of the capture characteristic time.

This result is very promising for bio-applications because it means that any surface potential changes caused by chemical or biological events can be registered with enhanced sensitivity by monitoring changes in capture time as a characteristic of a single trap.

\section{Conclusions}

To summarize, we fabricated new types of nanowires using complementary metal-oxide-semiconductor (CMOS) compatible top-down approach. Fabricated nanowire structures demonstrate pronounced RTS noise with advanced characteristics favorable for a new sensing approach based on single trap phenomena.

\section{Acknowledgements}

Silicon nanowire devices were fabricated using Helmholtz Nanoelectronic Facility (HNF) of Forschungszentrum Jülich. Y. Kutovyi greatly appreciates a research grant from the German Academic Exchange Service (DAAD).

\section{References}

[1] J. Li, S. Pud, M. Petrychuk, A. Offenhäusser and S. Vitusevich, Nano Letters 14 (2014), 3504-3509.

[2] N. Lukyanchikova, M. Petrychuk, N. Garbar, E. Simoen and C. Claeys, Appl. Phys. Lett. 73 (1998), 2444-2446.

[3] F. Gasparyan, I. Zadorozhnyi and S. Vitusevich, J. Appl. Phys. 117 (2015), 174506.

[4] M. Schulz, J. Appl. Phys. 74 (1993), 2649-2657. 